Relations industrielles

Industrial Relations

\title{
Le titre réservé de CRI et la déontologie de la profession
}

\section{Rodrigue Blouin}

Volume 42, numéro 2, 1987

URI : https://id.erudit.org/iderudit/050310ar

DOI : https://doi.org/10.7202/050310ar

Aller au sommaire du numéro

\section{Éditeur(s)}

Département des relations industrielles de l'Université Laval

\section{ISSN}

0034-379X (imprimé)

1703-8138 (numérique)

Découvrir la revue

\section{Citer cet article}

Blouin, R. (1987). Le titre réservé de CRI et la déontologie de la profession. Relations industrielles / Industrial Relations, 42(2), 309-324.

https://doi.org/10.7202/050310ar
Résumé de l'article

L'auteur interroge la déontologie de la profession, ses moyens de mise en œuvre et ses difficultés d'application.
Tous droits réservés @ Département des relations industrielles de l'Université Laval, 1987
Ce document est protégé par la loi sur le droit d'auteur. L'utilisation des services d'Érudit (y compris la reproduction) est assujettie à sa politique d'utilisation que vous pouvez consulter en ligne.

https://apropos.erudit.org/fr/usagers/politique-dutilisation/ 


\title{
Le titre réservé de CRI et la déontologie de la profession
}

\author{
Rodrigue Blouin
}

\author{
L'auteur interroge la déontologie de la profession, ses \\ moyens de mise en oeuvre et ses difficultés d'application.
}

Le contrôle de la compétence et du comportement des professionnels agissant en milieu du travail soulève d'innombrables difficultés. Dans la mesure où le professionnel est salarié, ses connaissances et aptitudes sont surveillées par l'entreprise ou le syndicat. Mais il peut néanmoins arriver que ce salarié soit confronté à des situations qui risquent d'enfreindre la déontologie. Tel est le cas par exemple lorsqu'un agent de gestion des ressources humaines est requis d'élaborer un test d'embauche discriminatoire. Il en va de même quand un conseiller syndical est confronté avec le fait de devoir déclarer une grève illégale. Quelles sont les règles de comportement alors applicables? Existe-t-il une protection spécifique contre les mesures disciplinaires motivées par le refus du professionnel d'obéir? Par ailleurs, de quelle protection bénéficie le public contre les incompétents qui agissent en pratique privée? Est-il possible par exemple d'empêcher un cancre de s'afficher à titre de négociateur ou de procureur?

La réforme du droit professionnel qui fut opérée en 1974 dans le but d'assurer une meilleure protection du public offre quelques éléments de solution à ces interrogations puisqu'elle consacre l'existence de la Corporation des conseillers en relations industrielles du Québec (CRI) ${ }^{1}$ comme la seule corporation habilitée au plan légal à surveiller la déontologie de ses membres. Cette question de la déontologie apparaît de nos jours d'une importance capitale alors que pullulent en milieu du travail de plus en plus d'associations professionnelles dont l'objet premier est avant tout l'intérêt de leurs membres. Force nous est en effet de constater que les corporations professionnelles si décriées il y a à peine quelques années gagnent actuelle-

* BLOUIN, R., professeur, Département des relations industrielles, Université Laval.

1 L'expression Corporation professionnelle des conseillers en relations industrielles apparaît dans le Code des professions, infra, note 3. Néamoins, la Loi créant la Société des conseillers en relations industrielles remonte à 1963 (S.Q. 1963, c. 99). Dorénavant, nous utiliserons dans l'exposé l'expression CRI. 
ment la ferveur d'une large clientèle avide d'obtenir un statut professionnel. On peut même se demander si nous n'aboutirons pas d'ici quelque temps à une situation analogue à celle qui prévalait antérieurement à la réforme et qui l'a motivée. À cette époque, il existait un phénomène prononcé d'accaparation des activités dites professionnelles par des groupes organisés. Or plus souvent qu'autrement, cette emprise s'était faite à l'ombre d'un puissant lobby corporatiste confondant souvent l'intérêt de la profession et celui du public. Aussi, la réforme des professions entreprise durant la période 1970-73, plus particulièrement en contexte de la santé, fut l'occasion pour le législateur de tenter un effort d'orientation'.

Par l'adoption du Code des professions $(\mathrm{CP})^{3}$, le statut de 38 corporations professionnelles fut précisé ou consacré4. Parmi celles-ci figure la CRI. Deux corporations ont depuis été reconnues ${ }^{5}$. Dorénavant, seules ces corporations, et celles créées éventuellement conformément à la loi, peuvent utiliser le titre de corporation professionnelle ${ }^{6}$. L'aire juridictionnelle de chacune est précisée et surtout ces corporations ne peuvent plus assumer la dualité de fonctions généralement poursuivies jusqu'à la réforme, à savoir protéger les intérêts de leurs membres et jouer le rôle de gardien de l'intérêt public; elles doivent désormais se limiter au second volet ${ }^{7}$. Ces corporations ne sont ni des syndicats, ni de simples associations professionnelles. Elles doivent surtout et avant tout s'assurer de la compétence et de la probité de leurs membres. Du régime alors institué, il sied de signaler pour les fins des présentes la création de l'Office des professions dont le rôle principal est de

2 Sur le sujet, consulter: R. DUSSAULT, et L. BORGEAT, «La réforme des professions au Québec», Revue du Barreau, 34, 1974, pp. 140-184. G. DUSSAULT, «L'évolution du professionnalisme au Québec», Revue Relations Industrielles, vol. 33, 1978, pp. 428-466. Office des professions du Québec, L'évolution du professionnalisme au Québec, Québec, 1976, $145 \mathrm{pp}$.

3 L.Q. 1973, c. 43 (devenue L.R.Q., c. C-26). Nous emploierons dans les pages qui suivent l'expression $\mathrm{CP}$ pour désigner le Code des professions.

$4 \mathrm{CP}$, supra, note 3, art. 24; cet article prévoit l'existence de 38 corporations professionnelles, dont 21 à titre exclusif et 17 à titre réservé. La CRI figure parmi ces dernières: voir tableau 1. Deux corporations se sont rajoutées depuis: voir, infra, note 5.

5 Il s'agit de la Corporation des technologues des sciences appliquées et de la Corporation des inhalothérapeutes.

6 CP, supra, note 3, art. 30. Sur le sujet: R. GAUDREAU, «L'exercice illégal des professions et l'usurpation des titres réservés», Revue générale de droit, 14, 1983, pp. 45-93.

7 CP, supra, note 3, art. 27. Ce n'est d'ailleurs qu'en assurant leur nécessité au nom de l'intérêt public que les corporations professionnelles pourront subsister. À ce propos G. DUSSAULT, «The Future of Professional Monopolies», Relations Industrielles, vol. 40, no 2, 1985, pp. 324-337. Aussi, du même auteur, "Les corporations professionnelles face au changement», Relations Industrielles, vol. 33, no 1, 1978, pp. 133-138. 
vérifier si les corporations professionnelles remplissent adéquatement leurs fonctions ${ }^{8}$ ainsi que celle du Tribunal des professions qui est un tribunal d'appel en matière disciplinaire'.

La loi constitutive de la CRI est, à toutes fins utiles, une loi professionnelle qui a pour but d'assurer la protection du public. Par ailleurs, elle permet tant au milieu syndical que patronal de se doter d'un personnel qui jouit du statut de professionnel au sens du CP. Mais le CP distingue deux sortes de professions: celles à titre exclusif et celles à titre réservé. Les conseillers en relations industrielles n'ont pas un champ de pratique exclusif. Un quidam peut donc exercer la profession sous d'autres titres et la CRI ne peut alors contrôler si celui-ci respecte les règles de la déontologie. Elle peut tout au plus le poursuivre pour usurpation de titre, si tel est le cas. Notre propos a pour objet de rappeler les axes fondamentaux du contentieux déontologique auquel sont assujettis les conseillers en relations industrielles. Est exclu de notre préoccupation le contentieux de l'usurpation des titres ${ }^{10}$. Nous abordons la question sous le seul prisme légal, c'est-à-dire en contexte de l'organisation professionnelle selon le $\mathrm{CP}$. Après avoir cerné la notion de déontologie, nous aborderons les moyens de mise en oeuvre et de surveillance pour terminer par une mise en situation des principales difficultés d'application du régime déontologique.

\section{LA NOTION DE DÉONTOLOGIE}

Au sens du Code des professions, les corporations n'existent que pour assurer la protection du public. Elles doivent à ce titre se préoccuper de la qualité des actes professionnels et du bon comportement de leurs membres. Cette philosophie de politique législative a favorisé l'émergence d'un droit professionnel dont l'axe fondamental est la déontologie, concept dont la compréhension renvoie à la notion d'activités professionnelles.

\section{L'activité professionnelle des conseillers en relations industrielles}

La notion légale de la profession témoigne d'une approche restrictive. Scrutée sous l'angle juridique, l'activité professionnelle s'entend en effet exclusivement de l'exercice d'une activité sous le couvert d'un statut profes-

8 CP, supra, note 3, art. 12 ss.

9 Le Tribunal fut déclaré inconstitutionnel: Crevier c. P.G.Q., (1981) 2 R.C.S. 220. La loi constitutive fut modifiée en conséquence: L.Q. 1982, c. 16.

10 Sur le sujet en général voir: R. GAUDREAU. «L'exercice illégal des professions et l'usurpation des titres réservés», supra, note 6. 
sionnel reconnu par le CP. Selon la loi, un professionnel est une personne qui détient un permis d'exercice délivré par une corporation et qui y est inscrite comme membre en règle ${ }^{11}$. Pour décider si une corporation professionnelle doit être reconnue, il est notamment tenu compte de l'ensemble des éléments d'appréciation suivants: les connaissances requises pour exercer l'activité concernée; les modalités d'exercice de l'activité et plus particulièrement le degré d'autonomie; le caractère personnel et confidentiel de la relation professionnelle; la gravité du préjudice subi par le fait de l'absence de contrôle sur la compétence et la probité des professionnels ${ }^{12}$. Une profession peut être à titre exclusif ou à titre réservé ${ }^{13}$; les premières se voient reconnaître un monopole quant au titre de la profession et quant aux activités professionnelles, tandis que les secondes ne bénéficient que du titre réservé même si leur constitution prévoit des activités définies. Le monopole d'exercice n'est reconnu que lorsqu'il ressort que la nature des actes posés et la latitude d'exercice sont telles que la protection du public exige que ces actes ne soient posés que par des personnes qui possèdent la formation et la qualification nécessaires pour devenir et demeurer membres d'un organisme qui surveille l'exercice de la profession. On trouvera au tableau 1 la liste des corporations actuellement reconnues.

Les conseillers en relations industrielles n'ont pas un champ de pratique exclusif; la loi ne protège que le titre et les initiales CRI ${ }^{14}$. La protection ne semble pas s'étendre aux titres équivalents comme par exemple, conseiller en relations du travail (crt). Il peut être opportun de rappeler que la loi n'autorise pas les conseillers en relations industrielles à s'attribuer le titre de procureur, de conseiller juridique ou tout autre titre analogue car ces titres sont réservés aux avocats et aux notaires ${ }^{15}$. Pour ce qui est de l'activité professionnelle d'un conseiller en relations industrielles, la loi constitutive la précise comme étant «exercer l'art d'établir, de maintenir et de modifier les relations entre employés, entre employeurs ou entre employeurs et employés» ${ }^{16}$. Cette définition reçoit dans le milieu diverses acceptions. Certains y voient un champ de pratique restreint aux relations du travail au sens le plus classique, d'autres embrassent une conception plus large qui comprend la gestion des ressources humaines et même, pour quelques-uns, l'administration des politiques publiques. Pour d'autres, la profession est essentiellement évolutive alors que quelques-uns estiment qu'il n'y a tout simple-

$11 \mathrm{CP}$, supra, note 3, art. $1 \mathrm{f}$ ).

12 Ibidem, art. 25.

13 Ibidem, art. 12, 26, 27, 36 et 37.

14 Loi sur la CRI, supra, note $1,11$.

15 Loi sur le Barreau, L.R.Q., c. B-1, art. 136 a); Loi sur le notariat, L.R.Q., c. N-2, art.

125. Sur le sujet, voir R. GAUDREAU, supra, note 6, en particulier ses références 86 et 96.

16 Loi sur la CRI, supra, art. 1; C.P, supra, note 3, art. 37 f). 
ment pas de champ de pratique caractéristique aux conseillers en relations industrielles. Mais cette controverse, si intéressante soit-elle, reste étrangère à la question de la déontologie. Car, quel que soit le champ d'activités reconnu aux conseillers en relations industrielles, s'il en est un, la CRI a essentiellement pour mandat légal «d'assurer la compétence et la probité dans l'exercice de la profession ${ }^{17}$. En fait, la déontologie est plus intéressée par le contexte d'exercice de l'activité professionnelle, quelle que soit sa portée, puisque, tel que nous le verrons ci-après, si le concept de faute déontologique trouve facilement application en contexte d'exercice d'une profession libérale, il en va autrement lorsque l'activité professionnelle est exécutée à titre de salarié.

Tableau 1

Corporations professionnelles

Titre exclusif

Agronomes

Architectes

Arpenteurs-géomètres

Audioprothésistes

Avocats

Chimistes

Chiropraticiens

Comptables agréés

Dentistes

Denturologistes

Infirmières et infirmiers

Ingénieurs

Ingénieurs forestiers

Médecins

Médecins vétérinaires

Notaires

Opticiens d'ordonnance

Optométristes

Pharmaciens

Podiatres

Techniciens en radiologie
Titre réservé

Administrateurs agréés

Comptables en administration industrielle

Comptables généraux licenciés

Conseillers d'orientation

Conseillers en relations industrielles

Diététistes

Ergothérapeutes

Évaluateurs agréés

Hygiénistes dentaires

Infirmières et infirmiers auxiliaires

Inhalothérapeutes

Orthophonistes et audiologistes

Physiothérapeutes

Psychologues

Techniciens dentaires

Technologistes médicaux

Technologues des sciences appliquées

Travailleurs sociaux

Urbanistes

17 Loi sur la CRI, supra, note 1, art. 3, 4 et 11. 
Le Code de déontologie de la Corporation des conseillers en relations industrielles

On reconnaît généralement un caractère sybillin au concept de déontologie, mais selon une vision purement juridique, la déontologie se limite à n'être qu'un corps de règles de droit positif qui précisent les devoirs du professionnel envers le public. Ces règles doivent se retrouver avant tout en un code de déontologie et chaque corporation professionnelle a l'obligation d'adopter un tel code ${ }^{18}$. Un code de déontologie n'est cependant, à toutes fins utiles, qu'un code de moralité professionnelle ${ }^{19}$ même si le caractère disciplinaire du régime déontolgique implique qu'il n'y a pas de faute déontologique sans texte justificatif. Il a en effet été observé que dans les codes de déontologie en vigueur «les textes constitutifs de la faute disciplinaire sont rédigés en des termes suffisamment généraux pour couvrir des situations non expressément définies et prohibées ${ }^{20}$ mais qui découlent de l'esprit de l'énoncé de prohibition d'un texte.

Aux termes du CP, les règles de tout code de déontologie doivent imposer au professionnel des devoirs d'ordre général et particuliers ${ }^{21}$. Ces règles doivent permettre de préciser la qualité de l'activité professionnelle et à ce titre elles doivent définir les activités, fonctions et actes dérogatoires à la profession. De plus, ces règles ont pour objectif d'encadrer la relation du professionnel avec ses clients et ses collègues, notamment de délimiter le secret professionnel. C'est pourquoi la CRI a adopté un tel code qui précise aux membres de la corporation des règles délimitant leurs devoirs et obligations envers "le public, le client et la profession»" ${ }^{22}$. Il y a au sein de ce code un bon effort de classement sous chacun de ces volets. Mais les règles qui y sont formulées n'apparaissent pas suffisamment claires et précises pour permettre au public et même au membre de se rendre compte à la lumière du libellé du texte de l'étendue du devoir ou de l'obligation. En cela, le Code de déontologie de la CRI rejoint bon nombre d'autres codes de même nature ${ }^{23}$ qui se limitent à circonscrire de façon large les actes dérogatoires ${ }^{24}$. Un examen des actes dérogatoires envers le public, le client et la profession nous permet de vérifier cette assertion.

18 CP, supra, note 3 , art. 12 a) et 87.

19 Techniciens en radiologie, (1980) D.D.C.P. 696, à p. 701 (Tribunal des professions).

20 Sur le sujet, voir: L. BORGEAT, «La faute disciplinaire sous le Code des professions", Revue du Barreau, 38, 1978, pp. 3-12.

21 CP, supra, note 3, art. 87.

22 Code de déontologie, A.C. 2454-77, 27 juillet 1977. Règlement 77-424, 29 juillet 1977. L'expression CD sera utilisée, dans les références, pour désigner le Code de déontologie.

23 Sur le sujet, on pourra lire: Y. OUELLET, «L'imprécision des codes de déontologie professionnelle», Revue du Barreau, 37, 1977, pp. 669-672.

24 L. BORGEAT, «La faute disciplinaire sous le Code des professions, supra, note 20. 
Les devoirs et obligations envers le public sont précisés au Code de déontologie en fonction de deux idées directrices. D'une part, le conseiller en relations industrielles doit exercer sa profession en respectant la dignité humaine ${ }^{25}$. À cet effet, il doit se préoccuper de la qualité du milieu du travail, de la promotion du travailleur et de la confidentialité des dossiers. D'autre part, le professionnel doit favoriser les mesures susceptibles d'améliorer la qualité des services par l'éducation, l'information et les travaux de recherche ${ }^{26}$. En ce qui concerne le client, les règles comportent un énoncé général précisant une obligation de comportement. Ainsi, le professionnel ne doit accepter de mandat que dans les limites de sa compétence, ce qui ne l'empêche pas de s'adjoindre des experts sur des sujets précis ${ }^{27}$. Il y a aussi des règles spécifiques qui visent la qualité des actes (intégrité, disponibilité, diligence), les conflits d'intérêts, le secret professionnel et les honoraires. Les énoncés sont pour la plupart à caractère général. Finalement, pour ce qui est des règles qui régissent le conseiller en relations industrielles envers sa profession, celles-ci visent la prohibition de moyens illégaux ou faux titres, l'interdiction de discrimination et l'obligation de contribuer à l'avancement de la profession ${ }^{28}$.

Ces diverses règles que l'on retrouve au Code de déontologie ou qui en découlent gouvernent l'exercice de la profession. Elles sont l'expression d'une approche générale qui valorise une idéologie de service à la société et qui préconise ce qui n'est somme toute qu'une morale professionnelle. Un manquement à l'une de ces normes peut constituer une faute déontologique et ainsi engendrer la responsabilité disciplinaire.

\section{LES MOYENS DE MISE EN OEUVRE ET DE CONTRÔLE DE LA DÉONTOLOGIE}

L'étude des moyens doit être replacée en contexte général de l'organisation et de la gestion de la profession, ne serait-ce que pour mieux situer les questions exclues du contentieux de la déontologie mais qui relèvent néanmoins du champ de compérence de la CRI ainsi que pour mieux rapprocher du phénomène sous considération d'autres moyens de pourvoi qui se fondent sur la responsabilité professionnelle. 


\section{L'organisation et la gestion de la profession}

De l'ensemble des organismes prévus au CP, c'est à la CRI que revient le rôle prédominant de voir à la gestion administrative et à la surveillance de la déontologie de ses membres. La CRI est par ailleurs assujettie au pouvoir de surveillance de l'Office des professions. Au sujet du rôle de la CRI, on doit distinguer les règles qui concernent l'accessibilité à la profession, question qui ne fait pas partie de la problématique de la déontologie, et les voies de surveillance de la compétence et de la probité. En ce qui a trait à l'adhésion à la corporation, il appartient à la CRI d'admettre les personnes qui rencontrent les conditions d'admissibilité à la profession ${ }^{29}$. Elle est de plus habilitée à émettre des certificats de spécialistes ${ }^{30}$, pouvoir qu'elle n'a pas exercé à ce jour. Pour ce qui est des voies de surveillance de la déontologie, le législateur a adopté une double approche: une préventive (l'inspection professionnelle) et une curative (le recours disciplinaire). À ces questions doit être rattaché un recours particulier, l'arbitrage des comptes (prévu au Code de déontologie).

Le régime de l'inspection professionnelle est à double volet: surveillance de l'activité et vérification des livres et registres. Pour ce qui est du premier aspect, le contrôle peut se concrétiser dans deux formes d'inspection: générale et particulière. L'une et l'autre sont effectuées par le Comité statutaire ${ }^{31}$. Elles se font à l'initiative soit du Bureau de la CRI soit de son Comité d'inspection ou encore, soit d'un membre. Ce régime appelle au moins deux observations: les critères et instruments destinés à mesurer la compétence restent flous; l'inspection doit respecter le droit du client au caractère confidentiel des informations livrées au professionnel en cette qualité. Dès qu'il y a inspection, un dossier est ouvert et il doit être tenu à jour par la suite. Ce dossier doit comprendre un résumé des qualifications et expérience du membre ainsi que l'ensemble des documents relatifs à l'inspection $^{32}$. Le professionnel a le droit de consulter son dossier et d'en obtenir copie $^{33}$. Le Comité peut conclure que le professionnel respecte les règles régissant la déontologie; il peut recommander la radiation; enfin, il peut estimer que le professionnel doit suivre un stage de perfectionnement. Quelle que soit la décision prise, il s'agit d'une recommandation au Bureau

29 CP, supra, note 3 , art. $40,42,46,86 \mathrm{~g}), 183$ et 184 .

30 Ibidem, art. 42. Loi sur CRI, supra, note 1, art. 10 b).

$31 \mathrm{CP}$, supra, note 3, art. 109 à 115 . Règlement sur la procédure du Comité d'inspection professionnelle, A.C., no 826-77, 16 mars 1977. Règlement 77-149, 18 mars 1977.

$32 \mathrm{CD}$, supra, note 22, art. 3.02. Règlement sur la tenue des dossiers et des cabinets de consultation, A.C. 663-77, 2 mars 1977. Règlement 77-93, 4 mars 1977.

33 À ce propos, voir: L. BERNIER, «L'inspection professionnelle et ses allures sybellines", Mc Gill Law Journal, 22, 1976, pp. 117-128. 
de la CRI qui possède le pouvoir décisionnel définitif. En ce qui a trait à l'inspection des livres et registres concernant l'activité professionnelle, elle vise à assurer que les dossiers comportent certaines données et qu'ils sont conservés en des lieux qui en assurent la confidentialité ${ }^{34}$. Si le conseiller en relations industrielles est salarié, ses dossiers sont conservés chez l'employeur ${ }^{35}$. Le Comité d'inspection peut faire diverses recommandations pour que les obligations prévues au Code de déontologie soient respectées, dont celle de radier temporairement le membre et de l'obliger à prendre durant cette période les mesures appropriées pour se conformer à ses obligations. Ces règles régissant l'inspection professionnelle sont demeurées un pur cadre de référence. Leur compréhension théorique soulève par ailleurs de nombreuses interrogations et, comme toutes les règles de même nature des diverses corporations professionnelles, leur application est source de difficultés, plus particulièrement dans la fonction publique ${ }^{36}$.

En ce qui concerne maintenant le régime disciplinaire, son fondement est toute infraction commise au Code des professions, ainsi qu'à la loi créant la CRI et aux divers règlements, plus particulièrement au Code de déontologie. Ainsi, lorsqu'un membre de la corporation a enfreint un devoir ou une obligation, le contentieux disciplinaire est susceptible d'être déclenché. Pour l'objet des présentes, il suffit de signaler l'existence d'un Comité de discipline créé en vertu du Code des professions et d'un syndic nommé parmi les membres de ce comité ${ }^{37}$. Le syndic peut à son initiative ou sur dénonciation écrite faire enquête et recommander qu'une plainte soit portée devant le Comité de discipline. L'instruction de la plainte devant ce comité est à huis clos (pour protéger la confidentialité des dossiers des clients) et obéit à la procédure contradictoire ${ }^{38}$. Plusieurs questions intéressantes se rapportent à la problématique de la nature et de la portée du régime. Il nous faut savoir si ce régime est de nature civile, pénale ou originale. À ce sujet, on admet généralement que le droit disciplinaire entretient une parenté avec le droit pénal ${ }^{39}$ bien que plusieurs dispositions législatives prévoient l'application de certains principes de procédure civile ${ }^{40}$. Puisque ce régime est de

34 CP, supra, note 3, art. 91 et 94 c) d).

35 Règlement sur la déontologie, supra, note 22, art. 2.02 et 2.07.

36 Sur le sujet, lire: R. DUSSAULT et G. PELLETIER, «Le professionnel fonctionnaire face aux mécanismes d'inspection professionnelle et de discipline institués par le Code des professions", Revue du Barreau, 37, 1977, pp. 2-90.

37 CP, supra, note 3 , art. 116 a 122 .

38 Ibidem, art. 137 à 150.

39 Sur la question, consulter: J.C. HÉBERT, «La responsabilité professionnelle en droit disciplinaire», Revue du Barreau, 1982, pp. 643-646. Technicien en radiologie, (1980) D.D.C.P. 696, a p. 699 (Tribunal des professions). Lambert c.Lippens (1976) C.A. 159, Béchard c. Roy, (1975) C.A. 509.

40 Par exemple, le Code des professions renvoie aux art. 139, 140, 157 et 165 du Code de procédure civile. 
nature quasi-pénale et que le pénal applique le principe "pas de faute sans texte», doit-on conclure qu'il ne peut y avoir de faute disciplinaire sans texte? À ce sujet, les auteurs sont d'avis que cette règle n'est pas appliquée en ce sens que le droit disciplinaire peut s'en tenir au principe de la liberté de qualification de la faute vu que les textes sont rédigés en termes généraux ${ }^{41}$. Enfin, comte tenu que le régime disciplinaire présume d'une faute, le Comité de discipline peut-il s'inspirer de certaines notions non expressément prévues au Code de déontologie ou dans les règlements, comme par exemple référer aux concepts de faute collective, de participation, etc.? Certains soutiennent qu'il ne peut $y$ avoir que faute individuelle et absence de complicité, de participation, etc. ${ }^{42}$. D'autres, auxquels nous nous rallions, sont d'avis «qu'il serait regrettable que la déontologie souffre de myopie en limitant la responsabilité disciplinaire à l'auteur principal du délit» ${ }^{43}$. En terminant, signalons qu'un appel à la décision du Comité de discipline est prévu au Tribunal des professions ${ }^{44}$; il semble que l'intervention est limitée aux cas où les comités de discipline «ont pris en considération des facteurs étrangers à l'accusation disciplinaire, ont fermé les yeux devant certaines circonstances atténuantes, ou ont exercé arbitrairement leur discrétion» ${ }^{45}$.

Reste finalement la procédure en conciliation et arbitrage de comptes. Ce sujet ne concerne la déontologie que par son inclusion au Code de déontologie. Aussi nous nous limitons à signaler l'existence de ce moyen mis à la disposition d'un client qui s'estime lésé par la facturation d'un conseiller en relations industrielles. Le mécanisme est en deux temps: conciliation du syndic puis intervention d'un Conseil d'arbitrage déclenchée par la signature d'un Acte de compromis ${ }^{46}$.

\section{Synopsis sur les autres moyens}

de pourvoi pour assurer la protection du public

Le régime disciplinaire fait-il obstacle à l'exercice de d'autres recours plus traditionnels? Rien n'autorise à conclure en ce sens si bien qu'il appert

41 Sur le sujet, voir supra les notes 23 et 24.

42 Voir: J.L. BEAUDOIN, «La responsabilité civile et pénale de l'employeur résultant de la violation des lois professionnelles», Revue du Barreau, 36, 1976, pp. 179-219.

43 Consulter: J.C. HÉBERT, «La prohibition des condamnations multiples en droit disciplinaire», Revue du Barreau, 42, 1982, pp. 461-466.

$44 \mathrm{CP}$, supra, note 3, art. 162 à 182. Sur le sujet: P. ISSALYS, «The Professions Tribunal and the Control of Ethical Conduct among Professionnals», McGill Law Journal, 24, 1978, pp. 588-627.

45 Y. OUELLET, «Le pouvoir de surveillance et de contrôle de la sévérité des sanctions disciplinaires et administratives", Revue du Barreau, 38, 1978, pp. 362-369.

46 Règlement sur la conciliation et arbitrage des comptes, A.C. 1164, 13 avril 1977, Règ. 77-206, 15 avril 1977. 
que la procédure instituée devant le Comité de discipline ne constitue pas un obstacle à une action civile ou à une poursuite pénale basée sur les mêmes éléments ${ }^{47}$. Bien qu'il ne nous appartienne pas ici d'élaborer sur ces régimes, il apparaît opportun d'observer que l'exercice en société de la profession soulève d'intéressants problèmes au plan de la responsabilité civile. Aussi, en matière de responsabilité contractuelle, le client de la société pourrait alléguer une obligation conjointe des membres en parts égales, mais non pas une obligation solidaire, sauf si elle est clairement stipulée au contrat de services professionnels ${ }^{48}$. Au plan de la responsabilité délictuelle, les règles veulent que pour engager les membres de la société, il faut démontrer la participation de chacun. Néanmoins, si une personne a passé un contrat avec un chef d'équipe, la responsabilité peut lui être attribuée pour n'avoir pas su organiser, coordonner, surveiller, tout comme elle peut être imputée à l'auteur du délit ${ }^{49}$. Il y aurait même, selon certains, émergence d'une notion de faute collective ${ }^{50}$, notion distincte de celle de faute commune au sens du Code civil ${ }^{51}$.

\section{LA DIFFICULTÉ D'APPLICATION DU RÉGIME DÉONTOLOGIQUE}

Il est difficile de s'interroger sur la qualité des règles qui concernent le régime disciplinaire auquel sont assujettis les membres de la CRI puisque le dépouillement des recueils jurisprudentiels des décisions disciplinaires ne nous a pas permis d'identifier des espèces concernant des membres de la profession. Par ailleurs, si l'on fait exception des cas de poursuite pour admission à la corporation sur la foi d'un faux diplôme, aucun jugement des tribunaux de droit commun n'intéresse le régime disciplinaire découlant du Code de déontologie. Enfin, il nous faut signaler que nous n'avons pu identifier aucune décision impliquant la responsabilité civile ou la responsabilité pénale d'un conseiller en relations industrielles en cette qualité. Par ailleurs, il faut être conscient qu'une corporation à simple titre réservé, comme la CRI, peut éprouver certaines difficultés en matière de contrôle de la déontologie. Car pour que la déontologie puisse s'appliquer, il faut qu'il y ait exercice d'une activité professionnelle par une per-

47 Consulter HÉBERT, supra, note 39.

48 Lire sur la question: F. FRENETTE, «La responsabilité civile des professionnels réunis en société de droit ou de fait», Cahiers de Droit, 17, 1977, pp. 617-626. Voir cependant les limites à la possibilité d'une action contre la société qui résultent des art. 12 de la Loi sur la CRI, supra, note 1, et de l'art. 3.03.07 du CD, supra, note 22.

49 Voir: F. DROUIN, F. BARAKETT et P.G. JOBIN, «La faute collective dans l'équipe professionnelle», Revue du Barreau, 38, 1978, pp. 49-78.

50 Ibidem.

51 Code civil, art. 1106. 
sonne qui s'identifie comme conseiller en relations industrielles. À ce propos, le Code de déontologie oblige les membres de la CRI à s'identifier comme tel ${ }^{52}$. On comprendra par ailleurs qu'il suffit au professionnel «pour se soustraire aux contrôles de la corporation, de se retirer de celle-ci et de continuer à exercer la profession, en toute impunité, sous d'autres titres que ceux réservés ${ }^{53}$. Cette donnée apparaît comme le justificatif essentiel invoqué par ceux qui préconisent l'octroi d'un champ de pratique exclusif pour la profession des conseillers en relations industrielles.

La solution du titre exclusif ne nous apparaît pas comme une voie heureuse, du moins non pas à titre de principe. D'autres moyens doivent être envisagés pour assurer un contrôle plus général sur la déontologie. Dans cette recherche, il nous faut avant tout tenir compte de la variété des tâches accomplies par les conseillers en relations industrielles ainsi que du contexte d'exécution des activités. Ả ce sujet, rappelons que les règles déontologiques ont pour objet d'assurer la protection du public, c'est-à-dire le bénéficiaire direct du service professionnel ou encore, la personne qui sera affectée par l'acte professionnel. Dans la mesure où l'activité professionnelle est exercée à titre de consultant (pratique privée), c'est-à-dire en relation directe avec le client, la déontologie professionnelle trouve son terrain normal d'application. L'activité professionnelle peut par ailleurs être exécutée en contexte d'un lien d'emploi. L'application de la déontologie est alors susceptible d'être confrontée avec diverses interférences dépendant du type de conflit qui peut exister entre le professionnel et son employeur alors que dernier doit assumer un service à offrir au public ou à un client. Il peut s'agir, entre le salarié membre de la CRI et son employeur d'un conflit dit professionnel, c'est-à-dire d'un conflit sur le contenu ou le contrôle de l'activité professionnelle (ex. le professionnel constate que le test d'aptitudes qu'on veut lui faire élaborer ou appliquer est discriminatoire). Généralement, le salarié tentera de faire primer la déontologie à moins qu'il ne trouve un intérêt de promotion de carrière ou économique à ne pas dénoncer la situation. Le second type de litige concerne le conflit dit institutionnel, c'est-à-dire lorsqu'il y a négociation sur les conditions de travail entre une association professionnelle ou syndicale qui représente le membre de la CRI et l'employeur. La déontologie peut alors être confrontée à la notion d'appartenance à un groupe de pression qui s'autorise d'une stratégie de négociation (par exemple, maintenir des services essentiels en cas de grève ou de simples services pour empêcher la détérioration des lieux de travail). Les valeurs définies par le groupe s'imposeront à la déontologie dès lors que la prédominance reconnue à cette der-

$52 \mathrm{CD}$, supra, note 22 , art. 3.01.06.

53 Sur le sujet, voir GAUDREAU, supra, note 6, p. 50. Voir aussi: Office des professions du Québec, Le titre réservé et la protection du public, 1982, $109 \mathrm{p}$. 
nière risque de nuire aux objectifs poursuivis. Par analogie, nous référons au fait qu'on a déjà observé que dans le réseau des affaires sociales «les valeurs syndicales l'emportent sur les valeurs professionnelles lorsque les syndiqués prennent conscience du fait que le maintien des services essentiels rend la grève inefficace» ${ }^{54}$. Finalement, il peut y avoir absence de conflit entre l'employeur et son salarié membre de la CRI et un contexte où la déontologie peut être opposée à la fidèlité à l'entreprise (ex. les cadres qui s'identifient à l'employeur lors d'une grève des syndiqués et qui refusent de respecter les dispositions anti-briseurs de grève ou encore, qui déclarent un lock-out illégal). La déontologie est alors minée par les intérêts dits de carrière. Le tableau 2 illustre les hypothèses de travail retenues.

Compte tenu de ce contexte, la revendication d'un champ de pratique exclusif ne nous apparaît pas, avons-nous déjà signalé, comme une panacée. En effet, l'octroi d'une exclusivité d'actes professionnels ne règlerait pas pour autant le problème de l'influence du milieu du travail sur le respect de la déontologie. Ce problème est le plus épineux parmi les difficultés qui confrontent actuellement la profession. On ne peut certes rejeter du revers de la main la question du titre exclusif. Admettons, par exemple, comme hypothèse de travail, que le groupe de travail sur les tribunaux administratifs retienne la qualité d'avocat comme condition d'accession aux tribunaux administratifs, que ce soit comme membre ou comme procureur. Il nous apparaît clair qu'en un tel cas la CRI devra réclamer un champ de pratique partagé pour ses membres avec les avocats en autant que les tribunaux du travail sont concernés. Mais dans la mesure où la seule question ici en cause concerne la déontologie comme raison d'être de l'exclusivité d'un champ de pratique, force nous est de constater qu'il n'y a pas de coïncidence normale et nécessaire entre ces deux phénomènes. La vitalité du régime déontologique se doit, à notre avis, de reposer avant toute chose sur un intérêt marqué et soutenu de la CRI pour ce sujet. Plus particulièrement, la corporation doit nouer des liens avec le milieu universitaire et les centres de formation professionnelle (syndicaux, patronaux, tiers intervenants) de façon à y dispenser des sessions de formation sur la déontologie. Elle doit aussi publiciser davantage son Code de déontologie et à ce propos, rappelons que ses membres ont l'obligation d'en laisser un exemplaire à la vue et à la disposition de leurs clients pour examen; pour faciliter cet objectif, la corporation devrait publier sa loi constitutive et le code en un format accessible. Un registre annuel des membres en règle devrait être publié. Ces mesures ne règleront cependant pas le problème résultant du subtil contrôle

54 Voir C. RONDEAU, «La déontologie professionnelle et l'action syndicale», Revue Relations Industrielles, vol. 38, no 1, 1978, pp. 139-141. Rapport à l'Office des professions du Comité d'étude concernant la déontologie professionnelle dans les conflits de travail, Québec, février $1978,48 \mathrm{p}$. 


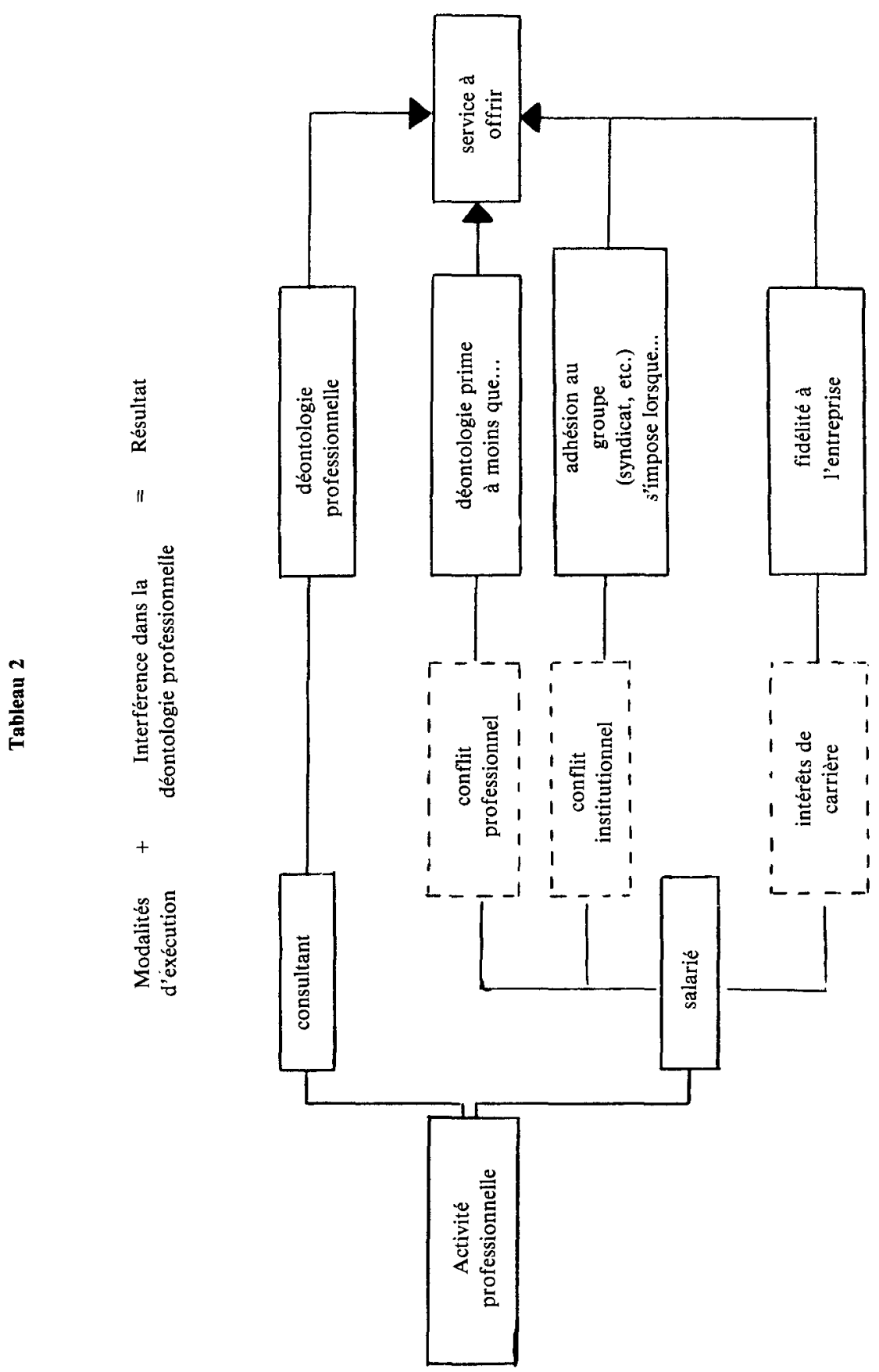


exercé par le milieu du travail sur la déontologie. C'est pourquoi, il apparaît nécessaire qu'une modification soit apportée à la loi constitutive de la CRI ou au CP. Le législateur devrait accorder une protection contre toute mesure disciplinaire ou administrative imposée à cause d'un refus d'obéir qui par ailleurs s'autoriserait du respect de la déontologie professionnelle. La protection devrait impérieusement s'appliquer pour les cas de refus d'embauche en raison du fait d'être membre de la corporation. La plainte pourrait relever du Comité de discipline avec appel au Tribunal des professions. Outre la réintégration, divers remèdes pourraient être prévus comme par exemple le paiement d'une indemnité pécuniaire punitive. De plus, les sanctions pénales devraient être réaffermies et atteindre les entreprises, les syndicats et les membres de la CRI qui participent à une infraction contre la déontologie ou complotent pour la commettre.

En définitive, la CRI est tenue de par la loi d'assujettir ses membres à des règles de compétence et de probité. Cette corporation est donc mieux placée que n'importe quelle autre association professionnelle pour apporter une contribution significative à la valorisation de la déontologie trop souventes fois ignorée dans un récent passé. En un tel contexte, il y va de l'intérêt de tous de collaborer à toute initiative que pourrait prendre la profession pour favoriser le développement de la déontologie.

\section{Professional Designation and Professional Ethics: The IRC}

In Québec, only associations created in conformity with the Professional Code may use the title of professional corporation. The PC admits two types of profession: those exercising exclusive jurisdiction over their designation and their field of activity, and those exercising exclusive jurisdiction over the former but not the latter.

The purpose of such corporations is to protect the public interest through their attention to the competence and professional conduct of their members. This approach to legislative policy has favoured the emergence of legislation regarding the professions the main thrust of which is professional ethics. The Corporation of Industrial Relations Counsellors (IRC) is a corporation in the second sense of the PC noted above.

The law in question defines the activities of Industrial Relations Counsellors as: «the art of establishing, maintaining and changing relations between employees, between employers or between employers and employees». The present author addresses himself to the questions of professional ethics, ways of application and difficulties of enforcement.

According to the terms of the PC, the professional corporation must adopt a code of ethics. The resulting code must require of the professional in question duties 
of both a general and a specific nature designed to cover activities, functions and inadmissible acts. The code of the IRC stipulates duties and obligations with regard to the public, the client and the profession. The concerning the public require of the Counsellor to exercise his profession with respect for human dignity. He must moreover, favour measures encouraging the quality of services through education, information and research. As regards the client, the Industrial Relations Counsellor can only accept a mandate falling within areas of his competence. Included also are specific rules dealing the quality of the professional act (integrity, availability and application), conflict of interest, confidentiality and fees. Obligations toward the profession involve the prohibition of discrimination and unbecoming behaviour.

The Corporation of Industrial Relations Counsellors must see to it that the code of ethics is respected. In turn, the Corporation is responsible before the Office des professions. The Corporation exercises its role through professional inspection, disciplinary measures and arbitration. A professional inspection committee is established. This committee can take measures to disbar a member, require him to enrol in a programme to improve and update his professional knowledge, or give directives concerning the keeping of records.

The disciplinary powers are of a semi-judicial nature. Each complaint is submitted to the Committee on discipline whose decisions are subject to appeal before the Tribunal des professions. In addition, a client convinced of unwarranted or excessive billing by a Counsellor can have recourse to conciliation by syndic followed by intervention of the Council for the arbitration of accounts.

As in the case of all other corporation, the Corporation of Industrial Relations Councellors is confronted with people who refuse to become members or decide to withdraw, in order to avoid its controle. At the same time, they continue to exercise their profession with impunity under another professional designation than that reserved for members of the Corporation. It is for this reason that there are a great number of demands for exclusivity of practice.

For the author, this solution does not seem to the most opportune, unless the working group on administrative tribunals reserves for lawyers the right of access to administrative tribunals as members or legal counsel. In this eventuality, it appears clear that the Corporation should demand a professional field shared by its members as well as those of the legal profession regarding labour courts.

It should be noted that the most serious threat to the profession originates in contexts where the Counsellor, acting as a salaried employee of a firm or union occasionally ignores professional ethics because of a supposed threat to the career. For this reason, the present author suggests that the law should be modified to provide protection against the use of administrative or disciplinary measures where the professional refuses to perform acts contrary to the code of ethics (for example: prepare a biased test, declare an illegal strike, refuse to hire Corporation members, etc.).

It is clear that the Corporation is required by law to subject its members to rules of competence and application. And it is in the best interests of the general public to encourage the initiative taken by the profession to further the development of a code of ethics. 